\title{
Biocatalytic Enzyme Networks \\ Designed for Binary-Logic Control of \\ Smart Electroactive Nanobiointerfaces
}

\author{
Marcos Pita ${ }^{a,},{ }^{*}$ Marina Privman ${ }^{b}$, Evgeny $\mathrm{Katz}^{c}$
}

\footnotetext{
${ }^{a}$ Instituto de Catalisis y Petroleoquimica, CSIC. C/ Marie Curie, 2. L10 28049 Madrid, Spain.

${ }^{b}$ Empire State College, SUNY, P.O. Box 908, Fort Drum, NY 13602-0908, USA

${ }^{c}$ Department of Chemistry and Biomolecular Science and NanoBio Laboratory, Clarkson University, Potsdam, New York 13699, USA

* Corresponding author: E-mail: marcospita@icp.csic.es
}

Keywords: Biocomputing, Biomolecular computing, Logic gate, Enzyme, Logic network, Interface, Biomedicine, Bioelectrochemistry

\begin{abstract}
Discussed are recent developments in biocomputing, outlining research progress from single enzyme-logic operations to systems of scaled-up complexity. Examples of specific logic operations are presented and strategies for system optimization to suppress the spread of noise in enzyme-logic networks are discussed. Special attention is given to electrochemical signal-transducing nanobiointerfaces, including ON-OFF switches and potential practical applications.
\end{abstract}




\section{Introduction}

By the end of the twentieth century, computers had become an essential tool in professional and personal lives of the members of technological societies. More recently, advances in information processing technology have inspired the development [1-3] of unconventional approaches to computing — information processing by means other than silicon-chip devices. Unconventional computing has been aimed at developing new applications and extending functionalities of electronic systems. One of the active research areas of unconventional computing is chemical computing, where Boolean logic operations are performed by using chemical reactions. A significant number of such operations have been realized with solutions of various chemicals $[4,5]$. Catalytic processes show particular promise for mimicking digital logic computing operations. Catalysts can function in a homogeneous environment, such as liquid solution, or localized at heterogeneous interfaces. Solid materials can be used as both support for a catalyst and signal transducer for the chemical information processing. Ultimately, miniaturization of heterogeneous chemical catalytic processes could reach single molecule computing operations implementing useful algorithms [6-8].

The emerging field of biocomputing utilizes the chemical reactions of molecules that are native to living organisms such as DNA or proteins. DNA computing [9-14] aims specifically at parallel information processing. Even though individual reactions involving complex DNA molecules take substantial time, this is compensated by massive parallelism of DNA information processing. As the result, for certain large computing tasks DNA systems could process information at a faster rate than microprocessors, which rely on fast sequential steps. Enzymes are proteins that catalyze biochemical reactions. These molecules are also commonly used in biocomputing [1518]. Enzymes are very specific with respect to the chemical reactions catalyzed by them: each interacts with only one particular reactant or class of reactants. Therefore, an enzyme can identify its specific reactant, or substrate, in a complex mixture of chemicals present in a living cell or artificial solution.

Approaches mimicking the circuitry of digital microprocessor using biomolecules have 
been explored. These include enzymes as elements needed to mimic digital logic operations [15-18]. For the near-term novel biomedical sensing and diagnostic applications, enzymatic reactions allow complex signal processing and analysis of different biomarkers for various kinds of injuries and other medical conditions. There is an ongoing research effort [19-21] to design signal-processing networks based on enzymatic reactions to process and correlate complex biochemical signals. It is aimed at addressing one of the most difficult tasks in biosensing: simultaneous analysis of biomarkers for multiple pathophysiological conditions. Such biomarkers can mask each other in complex mixtures or be individually indecisive for a particular condition.

In this work, we will discuss the progression from individual enzyme logic gates to their coupling into more complex enzymatic networks utilizing injury biomarkers as inputs. These systems rely on sensitive signal-transducing interfaces to communicate the output of enzymatic reactions as a YES/NO digital decision for presence of a pathophysiological condition and need for treatment.

\section{Enzymatic Biocomputing}

Binary logic operations, developed in nineteenth century by George Boole, serve as the foundation of digitalization and computing in informatics. In electronic devices, building blocks of digital circuits are logic gates. Each type of a logic gate is able to make a certain decision. Combinations of logic gates form circuits or networks, designed with specific tasks in mind. Digital operations include three elements: input(s), operator, and output(s) [22]. Inputs are initial values fed into to the system. They are transformed by a logic operator. Operators define the nature of a logic operation by setting a relationship to determine a specific final output for each input signal combination. Some more common examples of digital logic operators are AND, OR, XOR, NOT, NAND, NOR, InhA, and InhB. Results of digital logic operations can be expressed in the form of a truth table (Table 1) that describes the operator and correlates each combination of inputs with its corresponding output. Input and output values are always defined as $\mathbf{0}$ or $\mathbf{1}$. 
Mimicking digital logic operations with enzymatic reactions poses several challenges. First, each part of a biochemical reaction is to be identified with one of the three elements required to perform digital logic operation: input, output, and operator. The selection of chemical(s) for each one of the three elements is important, because this will determine how will the enzyme-logic gate (ELG) function. For example, substrate(s) of an enzyme may be identified as the input(s), one of the products as the output, and the enzyme itself as the machinery that constitutes the operator. It is also possible to define enzymes as inputs and their substrates as machinery, or to define machinery as comprised of both enzymes and non-protein molecules.

The second challenge for mimicking digital logic operations with enzymatic reactions is the transduction of the processed information, to convey the result of an enzyme-logic operation in a readily measurable form. To achieve this, physical and chemical changes occurring in enzyme-logic gates (ELGs) are linked to suitable transducing materials. An important direction in biocomputing has been the development of materials able to sense signals from biomolecular systems and act only when an enzyme-logic operation commands it. In electronics, the $\mathbf{1}$ and $\mathbf{0}$ input and output signals can be defined as the existence or the absence of electric current or voltage. In enzymatic computing, electric currents are replaced with changes in oxidation state, concentration, or physical properties of compounds participating in the enzymatic reaction. Some examples of measurable parameters the values of which might change over the course of a chemical reaction are $\mathrm{pH}$ of a solution, its optical absorbance, or temperature.

The third challenge in biocomputing is to assemble single logic operations into a network, where several ELGs are interconnected to allow more complex information processing. Since ELGs deal with chemical and physical changes, careful design of the network is required to avoid undesirable cross-talk between the interconnected elements of the network.

The fourth challenge involves digitalization and inclusion of correcting algorithms to minimize the chemical noise and processing errors resulting from operation of ELGs. It 
should be noted that many enzymatic reactions follow Michaelis-Menten kinetics, where reaction rates initially increase linearly with the substrate concentration. However, when concentration of a substrate approaches its largest possible (saturation) level, the graph of the reaction rate vs. substrate concentration reaches a plateau at the largest possible reaction rate. Substrate concentrations corresponding to the plateau can be associated with the value of $\mathbf{1}$ for an input signal. However, input signals with the value of $\mathbf{0}$, at zero substrate concentrations where the slope of the response curve is large, resulting in noisy output signals due to noise amplification. An important research effort [23] has been aimed at minimizing the spread of this noise.

Among the first single enzyme-logic operations to be developed, were AND, OR, XOR and InhB [24]. For all these ELGs, but the last one, the machinery is comprised of solutions of enzyme substrates and a dye precursor, such as ATBS, while solutions of the enzymes, which are present at high concentrations, serve as inputs. In the case of InhB, enzymes were immobilized to a glass support and other chemicals were used as inputs.

Several illustrations of enzyme-logic gates are provided below. Machinery of the AND gate discussed in [24] was defined as $100 \mathrm{mM}$ glucose and $100 \mu \mathrm{M}$ ABTS, dissolved in an aqueous oxygenated solution. Input signal A was enzyme glucose oxidase (GOx). Its input value $\mathbf{0}$ was defined as the absence of the enzyme. Input value $\mathbf{1}$ for GOx was defined as 0.15 enzyme activity units. Input B was microperoxidase (MP11). Its absence corresponded to the input value $\mathbf{0} ; 540 \mathrm{nM}$ concentration of MP11 was assigned the input value of 1. Adding GOx (input A) to the solution catalyzes oxidation of glucose (part of the machinery) and reduction of $\mathrm{O}_{2}$ to $\mathrm{H}_{2} \mathrm{O}_{2}$. MP11 (input B) catalyzes the oxidation of ABTS and reduction of $\mathrm{H}_{2} \mathrm{O}_{2}$. Oxidation of ABTS occurs only when input values for both GOx and MP11 are 1 (input combination 1,1). This corresponds to the AND logic operation. GOx is needed to produce $\mathrm{H}_{2} \mathrm{O}_{2}$ and MP11 is required to oxidize ABTS. Other three possible input combinations, 1,0, $\mathbf{0 , 1}$, and $\mathbf{0 , 0}$, corresponding to the absence of one or both enzymes, did not allow oxidation of ABTS to proceed. The oxidized form of ABTS served as the output signal. It is characterized by strong absorption at $\lambda=415 \mathrm{~nm}$ and is easily monitored spectrophotometrically. It should be 
noted that in order to assure the optimal performance of the system and work closer to the maximum possible reaction rate for GOx, glucose was used at much higher concentration than the range of its normal physiological values.

The OR gate, discussed in [24], was based on alcohol dehydrogenase (ADH) and glucose dehydrogenase (GDH). Its machinery was comprised of ethanol, glucose, and $\mathrm{NAD}^{+}$. The output of the system was $\mathrm{NADH}$, which is characterized by a strong absorption band at $\lambda=340 \mathrm{~nm}$. Addition of either one or both enzymes, ADH (Input A) and GDH (Input B), triggers oxidation of their corresponding substrates and reduction of $\mathrm{NAD}^{+}$to NADH. This corresponds to the OR logic operation. Thus, three of the four possible input combinations, $\mathbf{1 , 1}, \mathbf{1 , 0}$, and $\mathbf{0 , 1}$, result in the production of NADH corresponding to the output value of $\mathbf{1}$.

Logic operation XOR, produces output signal value $\mathbf{1}$ when one of the input signals is $\mathbf{1}$ and the other is $\mathbf{0}$. When both input values are $\mathbf{1}$, its output has to be $\mathbf{0}$. Mimicking this logic operation with enzymatic reactions was achieved using ADH and MP11 as inputs $A$ and $\mathrm{B}$, respectively. The machinery was comprised of ethanol, $\mathrm{H}_{2} \mathrm{O}_{2}, \mathrm{NADH}$ and $\mathrm{NAD}^{+}$. Variation in optical absorption by $\mathrm{NADH}$, measured at $\lambda=340 \mathrm{~nm}$, was defined as the output signal. Addition of $\mathrm{ADH}$ (input $\mathrm{A}$ ) to the working solution resulted in oxidation of ethanol and reduction of $\mathrm{NAD}^{+}$to $\mathrm{NADH}$, increasing concentration of $\mathrm{NADH}$. On the other hand, adding MP11 (input B) resulted in reduction of $\mathrm{H}_{2} \mathrm{O}_{2}$ accompanied by oxidation of NADH to $\mathrm{NAD}^{+}$, decreasing the concentration of NADH. When both inputs are added to the system, they balance each other in such a way that the ratio of concentrations of $\mathrm{NAD}^{+}$and $\mathrm{NADH}$ does not change above the threshold values.

In electronic systems, NAND and NOR are known as universal logic operations. It is possible to combine several NAND or NOR gates into any other existing digital logic operation [25]. Given their importance in informatics, mimicking NAND and NOR operations with enzyme-logic systems was considered an important advance for information processing based on enzymatic reactions [26]. NAND and NOR gates were developed by combining two modules. One of the modules is an AND or an OR gate. 
The other module is an Inverter. The purpose of an inverter is to convert the value of the output signal from $\mathbf{1}$ to $\mathbf{0}$ or from $\mathbf{0}$ to $\mathbf{1}$ (the NOT gate). Figure 1A shows the inverter based on three enzymes, ADH, MP11, and GOx, and their substrates, ethanol, NAD ${ }^{+}$, and oxygen. In this system, glucose served as an input and $\mathrm{NADH}$, monitored at $\lambda=$ $340 \mathrm{~nm}$, as an output. In the absence of glucose, input value $\mathbf{0}$, ADH catalyzes oxidation of ethanol and reduction of $\mathrm{NAD}^{+}$to $\mathrm{NADH}$, generating a positive output signal. When glucose is present in the working solution, input value $\mathbf{1}$, it is oxidized by GOx. This reaction also produces $\mathrm{H}_{2} \mathrm{O}_{2}$, which is subsequently reduced by MP11. The reduction of $\mathrm{H}_{2} \mathrm{O}_{2}$ is accompanied by oxidation of $\mathrm{NADH}$ back to $\mathrm{NAD}^{+}$, in quantities that overwhelm the activity of ADH. This inverter can be coupled with an AND or OR logic gate generating glucose as its output. The AND gate shown in Figure 1A is based on enzyme maltose phosphorylase (Mph) acting as the machinery, maltose as input A and phosphate as input B. Only when both inputs A and B are added to the solution, can Mph perform its function, converting each molecule of maltose into glucose and glucose-1-phosphate. The OR gate shown in Figure 1A utilizes two enzymes, invertase (Inv) and amyloglucosidase (AGS), as the machinery, and sucrose and maltose as inputs A and B, respectively. When only sucrose is added to the system (input combination $\mathbf{1 , 0}$ ), Inv produces glucose and fructose. When only maltose is added (input $\mathbf{0 , 1}$ ), glucose is produced by AGS. Adding both inputs simultaneously (input 1,1) also results in production of glucose.

The AND gate connected to the inverter in Figure 1A, constitutes the NAND gate. Figure $1 \mathrm{~B}$ shows high absorption by NADH when the AND gate does not generate glucose, i.e., for input combinations $\mathbf{0 , 0 , 0 , 1}$ and 1,0. Production of glucose by the AND gate for its input combination 1,1 results in the suppression of NADH signal. The OR gate connected to the inverter was able to suppress the NADH signal for all input combinations other then $\mathbf{0 , 0}$, Figure $1 \mathrm{C}$.

Another important result [26] was pairing NAND and NOR gates based on enzymatic reactions. Although doing so using digital circuitry, where both input and output signals are electric currents, is relatively straightforward, the task is more challenging for enzymatic reactions, where inputs and outputs are different chemicals. Development of 
an NADH-signal amplifier and of a converter able to utilize NADH as the input and produce glucose as the output was carried out as a proof of concept.

The next step in the development of biocomputing was to connect several enzyme-logic operations in series. This suggested the first practical application for an ELG system [27]. It was shown that concatenated AND gates can work as an enzymatic keypad lock. The system described in [27] consists of three AND logic gates, where output of the first gate serves as an input for the second one and the output of the second gate, as an input for the third one. Enzyme invertase (Inv) and sucrose served as inputs for the first AND gate. Adding both these inputs to the working solution results in production of glucose, which is the output of the first enzymatic reaction. The second AND gate is based on glucose, produced by the first reaction, and GOx. Oxygen dissolved in the solution acts as machinery. Given the presence of its two required inputs, the second gate produces $\mathrm{H}_{2} \mathrm{O}_{2}$ as its output. The third AND gate uses as its inputs $\mathrm{H}_{2} \mathrm{O}_{2}$ produced by the second gate and MP11. The output of the third enzymatic reaction is $\mathrm{ABTS}_{\mathrm{ox}}$, the oxidized form of ABTS. To assemble an enzymatic keypad lock, it is not sufficient to concatenate several logic operations. It is necessary to also establish the keypad "combination," encoded in the order in which the signals (inputs of the enzymatic reactions) are introduced. For this system, enzymes Inv, GOx, and MP11 were used as such inputs. When the cascade of their biocatalytic reactions was allowed to operate for $20 \mathrm{~min}$, the sufficient amount of $\mathrm{ABTS}_{\mathrm{ox}}$ to pass its threshold value was generated only when the three enzymes are added in the order in which they are listed above. When Inv, GOx and MP11 were added in any other possible order, the system failed to produce significant amounts of $\mathrm{ABTS}_{\mathrm{ox}}$. To mimic the level of complexity of a typical mechanical keypad lock with 10 input buttons, a keypad based on enzymatic reactions could utilize a series of additional "inert" chemical signals constituting the input value 0, entered in addition to the three input signals required to carry out the enzymatic processes described above.

It was shown that enzyme-catalyzed reactions can mimic not only logic gates, but also other circuitry components, such as Set-Reset (S-R) flip-flop memory unit [28]. An S-R memory unit allows switching back and forth between two states of the system, one of 
which is defined as Set and the other as Reset, while keeping the state unchanged when the original input is fed into the system. Such flip-flop systems should also be based on two interlinked outputs: when the Set output is $\mathbf{1}$, the Reset one must be $\mathbf{0}$, and vice versa. This behavior can be mimicked by an enzymatic system that activated one of its outputs while, at the same time, deactivated another one.

One example of such enzymatic system mimicking an S-R memory unit [28] utilizes enzyme diaphorase. This enzyme reduces NADH, resulting in the decrease of its optical absorption at $\lambda=340 \mathrm{~nm}$. At the same time, diaphorase oxidizes another redox mediator, 2,6-dichloroindophenol (DCIP). Glucose is selected as the Set input for this system. When added to the reaction mixture GOx oxidizes glucose. This generates $\mathrm{H}_{2} \mathrm{O}_{2}$, which is subsequently reduced to $\mathrm{H}_{2} \mathrm{O}$ by enzyme horseradish peroxidase (HRP). In this process, DCIP is converted from its reduced form, DCIP ${ }_{\text {red, }}$, to the oxidized one, DCIP $_{\text {ox }}$. Production of the DCIP ${ }_{\text {ox }}$ results in an appearance of its optical absorption band at $\lambda=655 \mathrm{~nm}$. The Reset input for the memory unit is ethanol. When added to the system, ethanol is oxidized to acetaldehyde. This reaction is catalyzed by ADH. At the same time, $\mathrm{NAD}^{+}$is reduced to $\mathrm{NADH}$, which results in the characteristic absorption band for NADH, at $\lambda=340 \mathrm{~nm}$. The function of diaphorase in this system is to erase the system's previous information, using part of the new output to change the oxidation state of the first marker. After establishing the proper balance between concentrations of $\mathrm{NAD}^{+}$and $\mathrm{DCIP}_{\text {red }}$, the system was able to flip-flop between its initial and final states several consecutive times.

Another enzymatic S-R memory circuit described in [28] was realized with an electrochemical output signal instead of an optical one. This system used ferrocyanide as the Set output signal instead of DCIP. Adding the respective Set or Reset signals to the reaction mixture allowed to switch between oxidative and reductive electric current. As a curiosity, the authors stored different memory units in a 96-well ELISA plate and performed a "computation," using each 8-well column as a character in ASCII code. Thus, they were able to overwrite the initially encoded word "Clarkson" with the word "University." The working solution, stored in a refrigerator, remained stable for weeks. 
Another important research effort has been aimed at digitalizing output and developing ability to consistently analyze noise in biocatalytic processes. Ideally, an output of a digital logic operation can have only two possible values, $\mathbf{0}$ and $\mathbf{1}$. However, mimicking such operations with enzymatic reactions results in a continuous measurable response, rather than in its discrete values. This problem can be circumvented by defining some arbitrary thresholds, for example, for maximum possible value for the $\mathbf{0}$ output and minimum possible value for the $\mathbf{1}$ output. All intermediate values between the two thresholds yield a non-determined output for a logic operation. Typically, an ELG's output is defined as the presence of a specific chemical or as a physical signal related to the presence of a chemical at certain concentration. Since enzymes are very selective and sensitive to the presence of their substrates, some noise in the output signal is produced even when a substrate is present at a level below its threshold for input value 0. Therefore, it is important to study the effect of noise on the performance of enzymelogic systems. Analysis and optimization of single [29] and concatenated [30] ELGs, noise reduction approaches [31], and search for sigmoidal response in enzymatic systems [32] has been reported. See also [23] for an extensive recent review.

One possible way to lessen the noise amplificiation by concatenated enzyme-logic reactions has been to develop chemical filters. This can be done, for instance, by using additional chemicals which can react with output signals, preventing the build up of their concentrations above $\mathbf{0}$, thus making the reaction output sigmoidal (with small slope at the origin) as a function of the input. An example of noise filtering and analysis of an Identity Gate operation, is described in [33]. The system employs $\mathrm{H}_{2} \mathrm{O}_{2}$ as an input, and enzyme HRP as the machinery, and a fixed quantity of redox mediator 3,3',5,5'-tetramethylbenzidine (TMB). The oxidized form of the mediator, $\mathrm{TMB}_{\mathrm{ox}}$, serves as the output, and its optical absorption is monitored at $\lambda=650 \mathrm{~nm}$. The filtering action is achieved by adding ascorbic acid, which reduces $\mathrm{TMB}_{\mathrm{ox}}$ to $\mathrm{TMB}$. The response at various reaction times ("gate" times), for different concentrations of both the input signal and the filtering chemical was studied. The analysis of the filter performance included determining a noise-scaling factor, which is related to concentration of ascorbic acid and the gate time. Determining the noise-scaling factor allowed to optimize the concentration of ascorbic acid and identify the best gate times 
for noiseless gate functioning.

One of the most commonly used ELG output signals is the change in $\mathrm{pH}$ of a working solution. As will be discussed below, this signal can be coupled with $\mathrm{pH}$-responsive polyelectrolytes [20]. A biochemical filter working with an output based on $\mathrm{pH}$ change has been developed [34]. When output of an ELG is a chemical that acidifies a working solution, then even at relatively low concentrations it might suffice to achieve the desired final value of $\mathrm{pH}$. In such systems, noise reduction plays a particularly important role. Taking into consideration that well-designed noise-suppressing filters for the binary input signals are characterized by sigmoid-shape response, a successful filter for an ELG should behave in a similar manner. It was shown that buffering a working biocatalytic solution results in such sigmoid output response. Developing the filter required to select a buffer with the suitable $\mathrm{pK}_{\mathrm{a}}$ and determine the appropriate concentration of the buffer for a given biochemical process. The system analyzed in [34] included ethyl butyrate as input, enzyme esterase (Est), and HEPES buffer. The initial buffer concentration was adjusted to yield $\mathrm{pH}$ 7. Est converts ethyl butyrate to butyric acid and ethanol. Protons released by dissociation of butyric acid are removed from working solution by HEPES. When the buffering capacity of HEPES is overwhelmed, the working solution begins to acidify and its $\mathrm{pH}$ decreases. These enzymatic processes were carried out using different concentrations of HEPES and ethyl butyrate, while activity of Est was fixed at 4 units $\cdot \mathrm{mL}^{-1}$. Experimental results provided clear evidence that, with proper optimization the output signal becomes sigmoid shaped, even though $\Delta \mathrm{pH}$ becomes less pronounced. Noise amplification analysis has shown that the maximum noise suppression value should be realized with approximately $250 \mathrm{mM}$ HEPES, for reaction times between 70 and $160 \mathrm{~min}$.

\section{Enzyme-Logic Gates Coupled to Sensor Materials}

ELGs based on different biocatalytic systems can be coupled with materials that sense and act upon changes in output signals. Two types of output signals suitable for this purpose, acting independently or in concert, have been widely experimented with: $\mathrm{pH}$ 
changes and electrochemical signals. ELGs used to control $\mathrm{pH}$ changes consisted of AND or OR logic operations combined with a Reset system [35]. One such AND gate employed sucrose and urea as machinery in an unbuffered solution and $\mathrm{Na}_{2} \mathrm{SO}_{4}$ as the supporting electrolyte. Enzyme GOx served as input A for this system and enzyme Inv as input $\mathrm{B}$. When the two inputs are added to the system in combination 1,1, a cascade of biocatalytic reactions converts sucrose into glucose and, subsequently glucose into gluconic acid. Gluconic acid acidifies the initially $\mathrm{pH}$-neutral working solution and, within several minutes, its $\mathrm{pH}$ decreases below 4 . The OR gate included ethyl butyrate, glucose, and urea as a machinery. Input A was GOx and input B - Esterase. Any of the possible input combinations triggered a biocatalytic process that produced an acid: gluconic acid, butyric acid, or both. This resulted in acidification of the working solution and the decrease in its $\mathrm{pH}$. The Reset system, employed in conjunction with both AND and OR logic operation, used urease (Ure) as the Reset input. Ure converts urea present in the machinery into ammonia. This raises $\mathrm{pH}$ of the working solution from its acidic value back to neutral or even slightly alkaline one.

The two AND and OR ELGs described above have been coupled to several $\mathrm{pH}$ sensitive materials [20]. The first such material was an alginate membrane, grafted on a conductive surface to construct electrode [35]. Atomic Force Microscopy (AFM) showed changes in porosity of the gel, and impedance spectroscopy showed changes in the permeability of the membrane on the electrode surface upon $\mathrm{pH}$ changes produced in situ by ELGs. These measurements confirmed the ability of alginate membrane to sense and respond to setting and resetting the signal controlled by the ELGs.

Another material that proved to be sensitive to changes in $\mathrm{pH}$ was polyvinylpyridine (PVP), grafted on silica nanoparticles, $200 \mathrm{~nm}$ in diameter [36]. Nitrogen atoms present in the PVP polymer chain residues can change their state of protonation in the $\mathrm{pH}$ range between 5.5 and 4.5. This $\mathrm{pH}$ range is compatible with the two AND and OR enzyme logic processes, described above, generating changes in $\mathrm{pH}$ as outputs. Both of these ELGs were used to control the state of PVP-silica nanoparticles. When the ELGs' output is $\mathbf{0}$ (no acid is generated), $\mathrm{pH}$ of a working solution is neutral. At neutral $\mathrm{pH}$, the polymer chains are deprotonated and hydrophobic, and the polymer layer shrinks. This 
allows PVP-covered nanoparticles to aggregate into clusters with diameter of about $3 \mu \mathrm{m}$. When the ELGs' output is $\mathbf{1}, \mathrm{pH}$ of the solution drops from about 6.5 to 4 , which is below the value of $\mathrm{pK}_{\mathrm{a}}$ for PVP. In acidic solutions, PVP chains get protonated. As a result, they become hydrophilic, the polymer layer swells, and nanoparticles repel each other and separate. Application of the Reset function based on Ure increased the $\mathrm{pH}$ of the working solutions back to slightly alkaline values, causing PVP to deprotonate and particles to reaggregate. The changes in silica particle size in response to the ELGs' output, were monitored with AFM and dynamic light scattering.

The AND and OR ELGs generating $\mathrm{pH}$ changes as their output signal were also used to control restructuring of Pickering emulsions. These are stabilized by microspheres modified with 3 polymers: a hydrophobic one (polystyrene), a hydrophilic one (polyethylether), and PVP. As discussed above, PVP changes from the hydrophobic to hydrophilic state and vice versa, depending on $\mathrm{pH}$ of a solution in which it is immersed [37]. The modified microspheres were immersed in a mixture of toluene and water at $\mathrm{pH}$ 6, forming water in oil (W/O) emulsion due to hydrophobicity of PVP at neutral $\mathrm{pH}$ values. When an ELG is provided with an input combination that results in output 1, pH decreases to 4 , within several minutes. In such mildly acidic solutions the emulsion changes its configuration to oil in water (O/W). ELGs' acid output was monitored by measuring changes in conductivity of the solution, which increases from about $100 \mu \mathrm{A}$ at $\mathrm{pH} 6$ to more than $2.5 \mathrm{~mA}$ at $\mathrm{pH}$ 4. Changes in the state of the emulsion were also monitored using a dye, 1,1,4,4-tetraphenyl-1,3-butadiene, which is soluble only in the organic phase.

An alternative way to design a logic system coupled to a signal-transducing material is to use substrates of enzymes as inputs, and enzymes - after their immobilization on silica particles - as machinery. Advantages of this method include possibility to reuse expensive enzymes. Using enzyme substrates as inputs may also facilitate future transition to flow processes for operating the system [38]. Machinery of the AND gate in [38] included two enzymes, Inv and GOx. Each enzyme was attached to aminofunctionalized silica spheres, $200 \mu \mathrm{m}$ in diameter, using glutaraldehyde as linker, Figure 2A. Input 1 was sucrose and input 2 - oxygen. Values $\mathbf{1}$ and $\mathbf{0}$ for input 1 were 
defined as a significant concentration of sucrose or its absence, respectively. Values for input 2 were defined as $\mathbf{1}$ for the natural concentration of $\mathrm{O}_{2}$ in solution in equilibrium with the atmosphere; and $\mathbf{0}$ as concentration of $\mathrm{O}_{2}$ remaining in solution after its deoxygenation by bubbling nitrogen gas for $20 \mathrm{~min}$. The AND gate output was gluconic acid, which was produced only for input combination 1,1, decreasing $\mathrm{pH}$ of the working solution. The OR gate machinery consisted of enzymes Est and GOx, also immobilized on silica particles, Figure 2B. Input 1 was ethyl butyrate and input 2 - glucose. The output of the system, causing decrease in $\mathrm{pH}$, was gluconic acid, butyric acid, or both acids. Reset was comprised of Ure immobilized on silica particles and its input signal urea. The output signal was transduced using magnetic nanoparticles covered with a thin layer of gold [39]. The gold-covered nanoparticles served as a scaffold for a selfassembly of a monolayer of mercaptopropionic acid, MPA, Figure 2C. When these nanoparticles are dispersed in a solution with neutral $\mathrm{pH}$, carboxylate groups of MPA are deprotonated and the particle surface acquires negative charge. Electrostatic repulsion between negatively charged particles conveys stability to the dispersion in $\mathrm{pH}$-neutral solutions. Upon the acidification of solution, as a result of ELG's activity, carboxylate groups become protonated and lose their charge. It is important to limit the decrease of $\mathrm{pH}$ in such systems to an appropriate level; when $\mathrm{pH}$ becomes too acidic, nanoparticles irreversibly aggregate. Measurements of dynamic light scattering allow determining whether such aggregation takes place. In addition to conveying stability to the dispersion, carboxylate groups of MPA also perform a sensing function. Their electrons contribute to the Localized Surface Plasmon Resonance (LSPR) signal of the gold layer. Protonation of carboxylate groups reduces electron density in the gold layer, and the LSPR absorption band shifts from $\lambda=530 \mathrm{~nm}$ to $\lambda=550 \mathrm{~nm}$, Figure 2D. Applying the Reset input to the system returned the LSPR signal to values close to the original ones.

Acid-producing AND and OR ELGs were also used to control the flat band potential of silicon chips. The chips, modified with thiol groups, were used as platform to immobilize gold nanoparticles modified with a self-assembled monolayer of MPA [40]. Controlled by ELGs, carboxylate groups of MPA protonated or deprotonated, causing a reversible change in the capacitance of the modified silicon chips. The shift in the chip 
capacitance served as the output of this system.

Among various materials used to create ELG-output transducing interfaces, PVP proved to be of particular interest. It can be grafted on conductive materials [41] and loaded with redox compounds, such as osmium bipyridine complexes, acting as mediators in enzymatic reactions [42]. Interfaces based on PVP were used to transduce $\mathrm{pH}$ changes generated by ELGs into electronic signals. Indium tin oxide, ITO, an electrically conductive and transparent material, emerged as a suitable support for PVP, for the purpose of constructing $\mathrm{pH}$-sensitive electrodes. The electrode [43] consists of a glass slide covered with a thin layer of ITO. ITO is functionalized with silane coupling agents, which covalently anchor polymer chains of PVP. When PVP is anchored using a derivative of epoxysilane, the polymer layer assumes configuration of a brush. In this state, the polyelectrolyte is single bonded to its support and its chains possess much higher mobility in solution than when the polymer is multiply bonded or absorbed on its support. The brush configuration proved to be particularly suitable for sensing changes in $\mathrm{pH}$. At $\mathrm{pH}>5.5$, $\mathrm{PVP}$ is in its deprotonated form, which is hydrophobic. The polymer layer shrinks and, as the result, a redox probe dissolved in the solution, ferrocyanide, is prevented from approaching the electrode surface to transmit electric signals. At $\mathrm{pH}<4.5$, the PVP brush is hydrophilic. It swells and becomes more permeable to the redox probe, permitting it to exchange electrons with the electrode surface. Ability of PVP to reversibly shrink and swell in response to $\mathrm{pH}$ changes in the solution provides the electrode with an external switch that operates under ELGs' control. The ON and OFF switching of PVP-modified ITO electrode with changes in $\mathrm{pH}$, has been successfully utilized to allow or prevent transmission of the signal from the ferrocyanide redox probe [43].

Once the signal transducing electrode was proved to sense and act in response to signals from an individual ELG, it has been coupled to a considerably more complex biocatalytic network. This network performed five logic operations and was based on three enzymes and four inputs, Figure 3A [44]. Two of its ELGs are connected in series. The first process is catalyzed by enzyme $\mathrm{ADH}$. One of its products, $\mathrm{NAD}^{+}$, is subsequently utilized by the second ELG. Since $\mathrm{NAD}^{+}$can be produced only in the 
presence of both input signals required by $\mathrm{ADH}(\mathbf{1}, \mathbf{1}$ input signal combination for $\mathrm{NADH}$ and $\mathrm{CH}_{3} \mathrm{COH}$ ), the first reaction corresponds to the logic AND gate. The second ELG, based on GDH, converts glucose into gluconic acid. Since the production of gluconic acid requires the presence of both glucose and $\mathrm{NAD}^{+}$, the GDH gate also realizes the AND logic operation. NADH produced as a by-product of the GDHcatalyzed reaction, recycles to the input of the first AND gate. This generates a feedback loop, Figure 3B, and permits the system to operate at low concentration of this input, below $1 \mathrm{mM}$. The latter is significant, because NADH's phosphate groups can buffer $\mathrm{pH}$ changes within the operation range of the biocatalytic network. Maintaining low concentrations of NADH keeps its buffering effect at non-appreciable level. The third logic AND gate, based on GOx, utilizes glucose and oxygen as inputs. Working in parallel with the first two ELGs, it also produces gluconic acid. "Signal splitting" of the glucose input between two parallel biochemical reactions models is the FANOUT function. Although FANOUT is very common in electronics, it is not as readily achieved in biochemical systems. The FANOUT gate was tuned by adjusting the activities of the GOx and GDH enzymes and, thus, the rates at which they consumed glucose. As a result, the two catalytic branches generated gluconic acid at about equal rates. Production of gluconic acid via two parallel reactions resulted in the logic operation OR. Using different input signal combinations to activate only the GDH or the GOx gate, or both, generated gluconic acid and the resulting drop in $\mathrm{pH}$ values. The acidic $\mathrm{pH}$ values could be reset to neutral values with urease and urea.

The 4 chemical inputs for the above network, $\mathrm{NADH}, \mathrm{CH}_{3} \mathrm{COH}$, glucose, and oxygen, can be added in 16 different combinations. Only five of these combinations result in the production of gluconic acid. Variation of $\mathrm{pH}$ with time was monitored following the application of each of the 16 input signal combinations. Input combinations that led to production of gluconic acid caused the $\mathrm{pH}$ values to drop below 4.5, within few minutes. Values of $\mathrm{pH}<4.5$ were defined as the output signal 1. Logic output 1 switched ON the ITO electrode modified with poly-(4-vinyl pyridine) (P4VP), Figure 3C and 3D. Ferro/ferricyanide mixture was used as the redox probe to measure the output signal. One of the electrochemical techniques used to analyze these electronic signals was cyclic voltammetry. Cyclic voltammogram recorded at the initially neutral 
$\mathrm{pH}$, when the interface is blocked and the electrochemical process is inhibited, resulted in a nearly flat curve. Similar flat curves were obtained for all combinations of the input signals which did not generate gluconic acid (output signal 0). Applying the five input combinations that generated gluconic acid and $\mathrm{pH}$ below 4.5 (output signal 1) resulted in current-voltage curves with two well pronounced peaks and high values of the peak currents. The biocatalytic resetting of $\mathrm{pH}$ to the initial, neutral value returned the interface to the OFF state and flat cyclic voltammogram.

Another system [45] took advantage not only of the PVP's ability to switch the signal transducing interface $\mathrm{ON}$ and OFF in response to commands from an ELG network, but also the fact that its pyridine residues can act as a linker for metal complexes [42]. In this sense, osmium bipyridine derivatives received particularly close attention, due to their ability to act as redox mediators for many different redox enzymes, remain covalently linked to PVP, and tune the formal potential of PVP by selecting an appropriate bipyridine derivative. Concentration of the Os complex bonded to PVP-functionalized ITO electrode can be adjusted, so that the Os-PVP system is active at $\mathrm{pH}$ of about 4, where the PVP layer is hydrophilic, swollen, and highly flexible. Flexibility of PVP chains allows electron transfer between Os complexes and ITO electrode. At $\mathrm{pH}$ 6, PVP is hydrophobic. In this state, the polymer layer shrinks, its chains are tethered and the electron transfer between different Os complexes and the electrode is inhibited. The redox potential of Os shifts with $\mathrm{pH}$, and its signal intensity increases with increasing acidity of the solution. The response of Os-PVP ITO electrode to changes in $\mathrm{pH}$ was monitored electrochemically, using AFM, and ellipsometry. The electrode was shown to reversibly open and close for several consecutive cycles.

When acidic $\mathrm{pH}$ opens the polymeric switch, Os-PVP ITO electrode, described above, could be connected to glucose oxidation biocatalyzed by GOx in the absence of $\mathrm{O}_{2}$. While connected to GOx, this electrode was also used in an enzymatic enable-reset system, which is similar to a flip-flop memory but utilizes only one output signal [46]. The enable input signal was ethyl butyrate and the machinery was Est. This biocatalytic process, produced butyric acid. Acidification of solution opened the polymer brush coupling the GOx redox process with the electrode. The reset input was urea, which was 
converted to ammonia in the process biocatalyzed by Ure. The resulting increase in $\mathrm{pH}$ closed the polymer brush, inhibiting the electron transfer between the enzyme and electrode.

As discussed above, modified switchable electrodes can readily transduce output signals generated by enzyme logic operations. Another important research efforts have coupled such electrodes with biocatalytic systems to control biofuel cells [47]. It has been shown that Os-PVP can mediate electron transfer required by the enzyme laccase to reduce $\mathrm{O}_{2}$ to $\mathrm{H}_{2} \mathrm{O}$ in a biofuel cell cathode. This switchable cathode was connected to an anode, where GOx biocatalyzed oxidation of glucose under anaerobic conditions. The biofuel cell was controlled by $\mathrm{pH}$-changing AND and OR logic gates and yielded the maximum power density of $0.7 \mu \mathrm{W} \cdot \mathrm{cm}^{-2}$ only when switched ON, by ELGs' biochemical inputs [48]. The switchable biofuel cell could also be controlled by a larger enzymatic logic network processing 16 possible input combinations, three of which generated output required to switch the cell on [49], as well as by an enzyme keypad lock concatenating three AND gates in the proper sequence to activate production of energy [50]. The ability to control biofuel cells using enzymatic reactions was further extended to antibody-antigen reactions [51]. As a proof of concept, the antibody-antigen switchable biofuel cell was shut down by HRP-catalyzed reaction, which reduces $\mathrm{H}_{2} \mathrm{O}_{2}$ while oxidizing a suitable substrate, such as ABTS. Addition of ABTS can cause an increase in $\mathrm{pH}$ from 4.5 to above 5.5, shutting down the biofuel cell. The support of the latter system was an ELISA plate with two different modified serum albumin proteins: a human serum albumin enhanced with dinitrophenyl (DNP) and a bovine serum albumin enhanced with nitrotyrosinase (NT). The system realized a NOR logic gate with goat Anti-DNP IgG as input A and rabbit Anti-NT IgG input B. When either one or both of the antibodies reach the support, they are immobilized on it. Secondary anti-goat and anti-rabbit IgG antibodies, loaded with HRP, are added as a revealing system. These are immobilized only if any of the primary antibodies are present.

\section{Approaching Enzyme Logic Operations under Physiological Conditions}


All of the systems discussed earlier operate in the acidity range, which is far removed from physiological conditions. Two strategies can be used to narrow the gap between the current working $\mathrm{pH}<5$ and the physiological $\mathrm{pH}$ 7. The first one is aimed at achieving a local $\mathrm{pH}$ change, in the vicinity of the electrode, while keeping $\mathrm{pH}$ in the bulk of the solution unchanged. The second approach is to find an alternative polyelectrolyte or copolymer that, unlike PVP undergoes a hydrophobic/hydrophilic transition at physiological $\mathrm{pH}$. The first approach is illustrated by the system where enzyme GOx, immobilized on magnetic nanoparticles, is concentrated on a switchable electrode surface [52]. Even in the presence of a mild buffer in the working solution, gluconic acid generated at the electrode surface can acidify an adjacent layer of the solution, without affecting the bulk $\mathrm{pH}$. A PVP-ITO modified electrode was used as a proof of concept. It was placed close to the wall of an electrochemical cell. A magnet outside the cell concentrated magnetic particles loaded with GOx at the electrode surface. The local $\mathrm{pH}$ change generated by formation of gluconic acid at the electrode surface could not be measured with a $\mathrm{pH}$-meter, which can only monitor $\mathrm{pH}$ in the bulk of a solution. The pH-dependent redox probe based on thionin was used instead. Thionin is able to penetrate a PVP layer in both its shrunken and swollen states. At the same time, thionin is characterized by a linear relationship between its normal redox potential and $\mathrm{pH}$. After calibrating the response of thionin to changes in $\mathrm{pH}$ by differential pulse voltammetry (DPV), it was possible to monitor simultaneously the changes in the bulk $\mathrm{pH}$, using a $\mathrm{pH}$-meter, and the local $\mathrm{pH}$ at the electrode surface, with thionin DPV. These measurements showed that, in the region adjacent to electrode surface, the $\mathrm{pH}$ decreased to about 5 , while the bulk $\mathrm{pH}$ remained at about 6.5. This constitutes a reasonable advacement towards biocompatibility of the operational $\mathrm{pH}$ range. Switching the electrode $\mathrm{ON}$ and OFF was demonstrated electrochemically, using ABTS as a probe. It was possible to detect its redox signal with cyclic voltammetry and impedance spectroscopy only when the local $\mathrm{pH}$ was decreased as the result of biocatalytic activity of GOx. The ABTS signal was not detected without the local acidification at the electrode surface. The system could be switched ON and OFF several consecutive times.

Another effort to control the local $\mathrm{pH}$ in the vicinity of PVP-ITO electrode has focused 
on immobilizing enzyme-loaded gold nanoparticles at a PVP thin-film [53], Figure 4A. Here, enzymes Est and Ure were immobilized, stepwise, on gold nanoparticles modified with carboxylate groups, which were adsorbed on PVP, Figure 4B. Upon addition of the respective substrates, ethyl butyrate and urea, the enzymes affect local $\mathrm{pH}$ changes. Addition of ethyl butyrate resulted in acidification and the consequent swelling of PVP. This allowed an external redox probe, such as ferrocyanide, to approach the electrode surface and generate an electrochemical signal, Figure 4C. When urea was added to the system, Ure produces ammonia and raises the $\mathrm{pH}$. This causes PVP to shrink, blocking the electrode surface and inhibiting the electrochemical signal.

As mentioned above, some effort has also been directed at developing polyelectrolytes or copolymers that transition between their hydrophilic and hydrophobic states at $\mathrm{pH}$ values closer to the physiological one. One such promising polyelectrolite is polyacrylic acid (PAA). When deposited on gold electrodes, it has been shown [54] to allow or inhibit the electrochemical signal of $\left[\mathrm{Fe}(\mathrm{CN})_{6}\right]^{3-/ 4-}$. Coimmobilization of PVP and PAA, $31 \%$ PVP-69\%PAA, has been reported [55]. The polymer mixture was grafted on ITO electrodes and used for selective gating to transport either anions or cations across the bipolymer film. The electrodes modified with this film performed better than the ones modified only with PVP. In the neutral $\mathrm{pH}$ range, PAA is hydrophilic. Its numerous organic functional groups are in carboxylate form, resulting in the negatively charged polymer surface. As $\mathrm{pH}$ becomes more acidic, carboxylate groups get protonated. As a result, the polymer surface gradually loses its negative charge and becomes hydrophobic. At the same time, acidification prompts protonation of pyridine groups of PVP. This has an opposite effect, making the electrode surface hydrophilic once again, but positively charged. The PVP-PAA properties have been characterized using AFM, ellipsometry, and contact angle measurements. AFM showed swollen configuration for $\mathrm{pH}<3$, when both PVP and PAA are protonated and the electrode is positively charged. For $\mathrm{pH}>6$, AFM revealed a rough configuration that corresponds to deprotonated PAA. For $\mathrm{pH} 4.5$, which was found to be an isoelectric point for this particular mixture of the polymers, AFM showed a smoother surface, which was attributed to the most compact configuration produced by the smaller proportion of charged functional groups in both polymers together with the positive-negative charge cancellation. Results of 
ellipsometry and contact angle measurements also demonstrated that at $\mathrm{pH} 4.5$, the thickness of the polymer layer is at its minimum value, and hydrophobicity is at its maximum. The PVP-PAA modified electrode was tested as an ion selective gate using two electrochemical probes: the negatively charged $\left[\mathrm{Fe}(\mathrm{CN})_{6}\right]^{3-14-}$ and the positively charged $\left[\mathrm{Ru}\left(\mathrm{NH}_{3}\right)_{6}\right]^{2+/ 3+}$. The electrode was $\mathrm{pH}$-titrated in the presence of both electrochemical probes, showing that upon acidification from $\mathrm{pH} 6$ to 3, the signal from the ruthenium complex diminishes while the ferrocyanide signal increases, intersecting at $\mathrm{pH}$ 4.4. Cyclic voltammetry was used to demonstrate the reversibility of the system. Only the appropriate redox probe was detected at each $\mathrm{pH}$ value tested: at $\mathrm{pH} 3$, where the polymer film surface positively charged, it was the negative $\left[\mathrm{Fe}(\mathrm{CN})_{6}\right]^{3-/ 4-}$, and for a negatively charged surface, at $\mathrm{pH} 6$, the positively charged $\left[\mathrm{Ru}\left(\mathrm{NH}_{3}\right)_{6}\right]^{2+/ 3+}$. PVP-PAA system was also proven to work as a 2-to-1 multiplexer, where two chemical input signals can be selected to give the same output signal. The output signal is detected as electron transfer resistance which can be measured by impedance spectroscopy; the redox probes $\left[\mathrm{Fe}(\mathrm{CN})_{6}\right]^{3-/ 4-}$ and $\left[\mathrm{Ru}\left(\mathrm{NH}_{3}\right)_{6}\right]^{2+/ 3+}$ act as the input signals and $\mathrm{pH}$ of the solution as the selector [56].

It has been shown that the PVP-PAA ITO modified electrode could also be operated by application of a constant potential [57]. The electrode was placed in a buffer solution

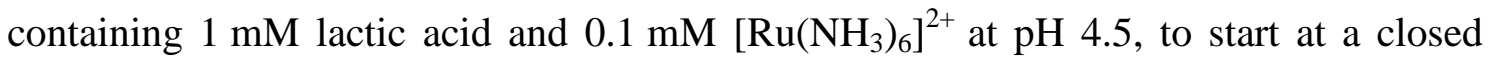
electrode configuration. Applying potential of $-0.85 \mathrm{~V}$ results in the reduction of oxygen at the electrode surface. This reaction consumes protons to produce both water and hydrogen peroxide. Cyclic voltammograms $(\mathrm{CVs})$ were recorded before and after the electrode polarization. They showed a transition from a no-signal CV to one where $\left[\mathrm{Ru}\left(\mathrm{NH}_{3}\right)_{6}\right]^{2+/ 3+}$ is clearly detected. The process was confirmed by simultaneous appearance of the reduction current due to consumption of oxygen. The local $\mathrm{pH}$ change was detected with differential pulse voltammetry measurements of thionin, the $\mathrm{pH}$-dependent electrochemical probe. After the electrode was polarized, the DPV signal of thionin shifted as a result of the local change in $\mathrm{pH}$, while $\mathrm{pH}$ in the bulk of the solution remained practically unchanged. When the electrode polarization was discontinued, diffusion restored the initial configuration of the system, making a reversible switch. 


\section{Biomedical Applications for Enzyme Logic Systems}

Examples discussed above illustrate significant progress in the development of biochemical computing systems and their coupling to different transducing materials. However, they represent only proofs of concepts. The majority of the biocomputing systems developed thus far, are activated by inputs that lack physiological relevance or require input concentrations that limit their biomedical applicability. Nonetheless, ELG networks show a great promise for applications as interfaces between natural physiological systems and artificial implantable biomedical devices, functioning as sensors and physiologically controlled actuators. For biomedical applications, such sensors should be able to accept and process biomarkers for various medical conditions as input signals and give binary output in the form of "Yes" or "No" response to the presence of a particular pathophysiological condition. This approach has been recently applied to the identification and analysis of proteins related to multiple sclerosis [58]. Enzyme-based logic gates can be used for simultaneous processing of multiple biomarkers for different injuries and signaling the presence of a specific injury. These systems take advantage of significant changes in concentration of specific biomarkers caused by physiological response to an injury. The use of injury biomarkers as input signals for ELGs in an AND-IDENTITY biocomputing system has been reported [59]. This work offers the first demonstration of ELGs' ability to discriminate among physiological concentrations for the selected biomarkers. The system, designed to process biochemical information that signals traumatic brain injury (TBI) and hemorrhagic shock (HS), is based on the synchronized operation of three enzymes, GDH, HRP and Lactate Oxidase (LOx), Figures 5A and 5B. Biomarkers for these conditions, glucose, lactate, and norepinephrine (NE), served as the input signals. The $\mathbf{0}$ input values correspond to normal physiological levels for the selected biomarkers, $4 \mathrm{mM}$ for glucose, $2 \mathrm{mM}$ for lactate and $2.2 \mathrm{nM}$ for NE. The $\mathbf{1}$ input value correspond to considerably higher concentrations of biomarkers which signal an injury. Concentration of $\mathrm{NE}$ in the blood is increased up to $3.5 \mu \mathrm{M}$ as the result of either TBI or HS. Concentration of lactate in the blood is increased to $13 \mathrm{mM}$ when either one or both HS 
and TBI injuries take place. The increase in glucose concentration to $30 \mathrm{mM}$ signals HS. The AND gate employs two enzymes, LOx and HRP, and two inputs, lactate and NE. Only when both inputs are $\mathbf{1}$ the output signal, norepi-quinone (NQ), is produced in significant amounts, Figure 5C. NQ can be detected spectroscopically at $\lambda=465 \mathrm{~nm}$ or chronoamperometrically, at $\mathrm{E}=-0.25 \mathrm{~V}$ vs. $\mathrm{Ag} / \mathrm{AgCl}$. The IDENTITY gate employs $\mathrm{GDH}$ and $\mathrm{NAD}^{+}$, and its only input is glucose. The appearance of glucose allows the reduction of $\mathrm{NAD}^{+}$to $\mathrm{NADH}$ which is detected spectroscopically at $\lambda=340 \mathrm{~nm}$ or chronoamperometrically at $\mathrm{E}=0.75 \mathrm{~V}$, Figure 5D. The two outputs of the ANDIDENTITY system, NQ and NADH, serve to identify the nature of the injury. For the input pattern defined as glucose, lactate, NE, the input combination $\mathbf{0 , 1 , 1}$ yields an output signal combination $\mathbf{1 , 0}$, signaling the occurrence of TBI. The input combination 1,1,1 yields the output 1,1 and corresponds to HS.

To date, research efforts aimed at increasing the complexity of biocomputing systems capable of analyzing various injury patterns based on their biomarkers, have followed two directions: multiplexing several ELGs in parallel and networking the ELGs. The network approach has been successfully used to distinguish between Soft Tissue Injury (STI) and TBI, by processing ten biomarkers used as input signals. This provided a higher degree of confidence than a system processing only one or few biomarkers [60]. The effectiveness of more complex networks, trying to correlate large number of specific biomarkers through several enzymatic steps to process the inputs utilizing more enzymatic reactions to achieve a definitive output signal, is limited by cross-talk of the reactions and noise amplification. Muptiplexing ELGs helps to overcome some of the limitations imposed by complex networks. This parallel approach has been successfully applied to develop a diagnostic method for simultaneous analysis of biomarkers for multiple injuries and identify each injury in the form of a binary Yes/No response [61]. This example, focused on six injuries: Soft Tissue Injury, Traumatic Brain Injury, Liver Injury, Abdominal Trauma, Hemorrhagic Shock and Oxidative Stress. A six-digit output marked each injury via AND or NAND ELGs, processing injury-specific biomarkers. Some of these ELGs have been also successfully tested in human serum [62]. It is noteworthy that this multiplexing system resembles a modular approach with flexible possibilities for identifying different injuries, which adds value to its 
commercial applicability.

Similarly to ELGs developed as a proof of concept, the ones intended for biomedical applications can also be coupled to signal-responsive materials for assessing a response to an input processed by the logic system. P4VP-ITO electrode has been successfully tested as an electrochemical transducer for the AND logic system detecting liver injury [63]. The logic system's machinery was comprised of enzyme GDH, and chemicals alanine, $\alpha$-ketoglutarate, glucose, and ferrocyanide in a $1.2 \mathrm{mM}$ Tris-buffer solution. Two enzymes that act as biomarkers for liver injury, Alanine Transaminase (ALT) and Lactate Dehydrogenase (LDH) serve as the inputs. Concentrations of the inputs present under normal or pathophysiological conditions were selected as input values $\mathbf{0}$ and $\mathbf{1}$, respectively, Figure 6A. The output of the system was defined as decrease in $\mathrm{pH}$, caused by the oxidation of glucose to gluconic acid. Acidification of the working solution opens the P4VP-ITO electrode and allows to detect the presence of electrochemical probe, $\left[\mathrm{Fe}(\mathrm{CN})_{6}\right]^{4-}$, the Figure $6 \mathrm{~B}$ and $6 \mathrm{C}$. The four possible input combinations were tested, and the input $\mathbf{1 , 1}$ was the only one able to trigger the whole biocatalytic cascade which included the following reactions. Addition of input 1, ALT, catalyzes the transformation of $\alpha$-ketoglutarate into glutamate, while producing pyruvate from alanine. Adding Input 2, $\mathrm{LDH}$, results in the reduction of pyruvate to lactate and oxidation of $\mathrm{NADH}$ to $\mathrm{NAD}^{+}$. In the presence of $\mathrm{NAD}^{+}, \mathrm{GDH}$ catalyzes oxidation of glucose, generating gluconic acid. The resulting decrease in $\mathrm{pH}$ switches on the electrode and opened it to electrochemical reaction of $\left[\mathrm{Fe}(\mathrm{CN})_{6}\right]^{4-}$. Behavior of the electrode in its ON and OFF state was characterized using cyclic voltammetry and impedance spectroscopy.

\section{Conclusions and Perspectives}

Chemical catalysis performed by enzymes has found a new niche of application related to computing technologies. In particular, enzyme-catalyzed chemical reactions have been successfully utilized to mimic various computing logic elements, where substrates and enzymes can perform either as logic machinery or input signals and the products 
provide the output signal. The level of complexity in biocomputing increased from single-enzyme logic gates, such as AND, OR, XOR, NAND, and NOR, to networks carrying out several concatenated logic operations and using multiple enzymes as inputs or machinery of enzyme logic gates. Significant effort has been devoted to optimization and suppression of noise in enzyme logic systems. The output signals generated by the enzyme logic operations were initially detected as a spectroscopical change and later on connected to electrochemical devices. Extensive work was performed in the area of switchable electrode interfaces by modification of electroactive surfaces with signalresponsive materials such as $\mathrm{pH}$-dependent polyelectrolytes. The design of enzymelogic systems able to produce an acid or a base yielded outputs in the form of $\mathrm{pH}$ that could change the polyelectrolytes from isolating to conducting form, thus allowing the transduction of chemical signals processed by biocatalytic reactions into electrochemical ones. The combination of ELGs and pH-switchable electrodes was successfully used to process biochemical information and generate a YES or NO decision that triggers a command for specific action. Extension of the biocomputing control of switchable electrodes has also addressed the switching ON and OFF of biofuel cells producing electrical power on-demand depending on physiological conditions; it might also be extended to sense-and-act biomedical systems designed to detect and treat injuries received as a result of polytrauma.

Future development of enzyme logic networks may occur in several directions. The flexibility of enzyme reactions allow for many potential networks to respond the needs of specific analytical problems that go beyond the enzymatic models, for pathogen search that mark different injuries or diseases in any living system, for those interesting for agriculture and farming common diseases or toxins. Miniaturization of the potential devices is also another development direction that would help to increase the response signal in terms of time response, reliability and signal-to-noise ratio. Finally, the finding of new transducing systems other than $\mathrm{pH}$ changes is a very important development direction that will help the potential application of enzyme biocomputing in new biorelated research areas.

\section{Acknowledgements}


Dr. M. Pita acknowledges the Ramon y Cajal 2009 program from the Spanish MICINN. Prof. E. Katz acknowledges research funding by the US-NSF (grant CCF-1015983). 


\section{Figure Captions}

Figure 1. (A) Schematic representation of the three different modules that comprise the NAND and the NOR logic gates: the AND module with Mph, the OR module with Inv and AGS, and the Inverter module with alcohol dehydrogenase, MP11 and GOx. (B) NAND spectroscopic results for different maltose and phosphate input combinations monitored at NADH absorption band centered at $\lambda=340 \mathrm{~nm}$. (C) NOR spectroscopic results for sucrose and maltose input combinations monitored at NADH absorption band centered at $\lambda=340 \mathrm{~nm}$. Reprinted with permission from J. Phys. Chem. B 2009, 113, 16065-16070, Copyright 2009 American Chemical Society.

Figure 2. (A) AND-Reset enzyme logic system capable of changing the $\mathrm{pH}$, comprised of the enzymes GOx and Inv, for the AND gate, and Ure for the Reset. (B) OR-Reset enzyme logic system capable of changing the $\mathrm{pH}$, utilzing the enzymes GOx and Est for the OR gate, and Ure for the Reset. (C) Transducing system based on gold-covered magnetic nanoparticles modified with 3-mercaptopropionic acid. (D) Surface Plasmon Resonance band shift resulting from operation of the OR gate. Reprinted with permission from ACS Nano, 2008, 2 (10), pp 2160-2166, Copyright 2008 American Chemical Society.

Figure 3. (A) Biocatalytic cascade used for logic processing of chemical input signals and producing in situ $\mathrm{pH}$ changes as the output signal. (B) Equivalent circuit for biocatalytic cascade. (C) Sample cyclic voltammograms of $\left[\mathrm{Fe}(\mathrm{CN})_{6}\right]^{4-}$, obtained for the ITO electrode modified with the P4VP polymer brush in (a) the initial OFF state, $\mathrm{pH}$ ca. 6.7; (b) the ON state enabled by the input combination $\mathbf{1 , 1 , 1 , 0}$, recorded at $\mathrm{pH}$ ca. 4.3; and (c) in situ reset to the OFF state, $\mathrm{pH}$ ca. 8.8. (Inset) Step 1, initial OFF state; step 2, ON state; step 3, reset to the OFF state. (D) Anodic peak currents, $I_{p}$, for the 16 possible input combinations. Dotted lines show threshold values separating logic 1, undefined, and logic $\mathbf{0}$ output signals. Reprinted from Reference 42 with permission, Copyright Royal Society of Chemistry (2009). 
Figure 4. (A) Reversible switching ON-OFF of an electrochemical reaction by biocatalytic processes at a functionalized interface. (B) Assembling of a switchable biocatalytic nanostructured interface. (C) Cyclic voltammograms obtained on the modified ITO-electrode in the presence of $\mathrm{K}_{4}\left[\mathrm{Fe}(\mathrm{CN})_{6}\right]$, upon application of chemical signals. (Upper) Bulk pH = 6: (a) before, and (b) 30 min after the application of ethyl butyrate. (Lower) Bulk $\mathrm{pH}=4.5$ : (a) before, and (b) $30 \mathrm{~min}$ after the application of urea. Arrows show the direction of the biocatalytically induced changes. Chem. Comm. 2010, 46, 2088 - 2090, Reproduced by permission of The Royal Society of Chemistry (2010).

Figure 5. (A) Biochemical reactions catalyzed by LOx and HRP for the AND gate, and GDH for the Identity gate with the equivalent logic systen used for processing the lactate, norepinephrine and glucose input signals. (B) Truth table for the eight possible input combinations. (C) Bar diagram featuring the combined AND-IDENTITY logic operation of the optical $(\lambda=465 \mathrm{~nm})$ and electrochemical $(\mathrm{E}=-0.25 \mathrm{~V})$ systems detecting the norepi-quinone output. (D) Bar diagram featuring the combined ANDIDENTITY logic operation of the optical $(\lambda=340 \mathrm{~nm})$ and electrochemical $(\mathrm{E}=0.75 \mathrm{~V})$ systems detecting the NADH output. Reprinted from Biosensors and Bioelectronics 2009, 24, 3569-3574 with permission, Copyright 2009 Elsevier.

Figure 6. (A) Biocatalytic cascade used for the logic processing of the chemical input signals, causing in situ $\mathrm{pH}$ changes and activation of the electrode interface. (B) $\mathrm{pH}$ changes generated in situ by the biocatalytic cascade activated with various combinations of the two chemical input signals, ALT, LDH: (a) 0,0; (b) $\mathbf{0 , 1}$; (c) $\mathbf{1 , 0}$; (d) 1,1. The dotted line corresponds to the $\mathrm{pK}_{\mathrm{a}}$ value of the PVP brush. (C) Cyclic voltammograms obtained for the P4VP-ITO electrode in (a) the initial OFF state at $t=$ 1 min, pH 6.3, and (b) the ON state enabled by the ALT, LDH input combination 1,1, at $\mathrm{t}=80 \mathrm{~min}, \mathrm{pH}$ 4.75. Reprinted with permission from ACS Appl. Mater. Interfaces 2011, 3, 1620-1623, Copyright 2011 American Chemical Society.

Table 1. Truth tables for different Boolean logic operations. 


\section{References}

[1] C. Teuscher and A. Adamatzky (eds.), Unconventional Computing 2005: From Cellular Automata to Wetware, Luniver Press (2005).

[2] A. Adamatzky and C. Teuscher (eds.), From Utopian to Genuine Unconventional Computers, Luniver Press (2006).

[3] Y. M. Yin and X. Q. Lin, Prog. Chem. 13 (2001) 337.

[4] A. Adamatzky, B. De Lacy Costello and T. Asai, Reaction-Diffusion Computers, Elsevier Science (2005).

[5] A. Adamatzky, Computing in Nonlinear Media and Automata Collectives, Taylor \& Francis (2001).

[6] G. Bell and J. N. Gray, Beyond Calculation: The Next Fifty Years of Computing, edited by P. J. Denning and R. M. Metcalfe, Copernicus/Springer (1997), Chap. 1, p. 30.

[7] T. Sienko, A. Adamatzky, N. G. Rambidi, and M. Conrad (eds.), Molecular Computing, MIT Press (2005).

[8] A. Adamatzky, IEICE Trans. Electron. E87C (2004) 1748.

[9] L. M. Adleman, Science 266 (1994) 1021.

[10] G. Paun, G. Rozenberg, and A. Salomaa, DNA Computing-New Computing Paradigms, Springer (1998).

[11] Z. Ezziane, Nanotechnology, 17 (2006) R27.

[12] N. Jonoska, J. Computer Sci. Techn. 19 (2004) 98.

[13] L. Kari, G. Gloor, and S. Yu, Theoretical Computer Science 231 (2000) 192.

[14] J. Watada, S. Kojima, S. Ueda, and O. Ono, Int. J. Innov. Comp. Inf. 2 (2006) 273.

[15]R. Baron, O. Lioubashevski, E. Katz, T. Niazov, and I. Willner, Angew. Chem. Int. Ed. 45 (2006) 1572.

[16]R. Baron, O. Lioubashevski, E. Katz, T. Niazov, and I. Willner, Org. Biomol. Chem. 4 (2006) 989.

[17]R. Baron, O. Lioubashevski, E. Katz, T. Niazov, and I. Willner, J. Phys. Chem. A 110 (2006) 8451.

[18]T. Niazov, R. Baron, E. Katz, O. Lioubashevski, and I. Willner, Proc. Natl. Acad. USA 103 (2006) 17160. 
[19]M. Pita and E. Katz, J. Comput. Theor. Nanos. 8 (2011) 401.

[20]S. Minko, E. Katz, M. Motornov, I. Tokarev and M. Pita, J. Comput. Theor. Nanos. 8 (2011) 356.

[21]J. Wang and E. Katz, Anal. Bioanal. Chem 398 (2010) 1591.

[22]G. Boole, An Investigation of the Laws of Thought. Macmillan Publishers Ltd. (1854). Reprinted with corrections, Dover Publications, New York, NY, 1958.

[23]E. Katz and V. Privman. Chem. Soc. Rev. 39 (2010) 1835.

[24]G. Strack, M. Pita, M. Ornatska and E. Katz. Chem. Bio. Chem. 130 (2008) 1260.

[25]S. G. Shiva, Introduction to Logic Design, 2nd ed.; Marcel Dekker, New York, 1998.

[26]J. Zhou, M. Arugula, J. Halámek, M. Pita and E. Katz. J. Phys. Chem. B 113 (2009) 16065.

[27] G. Strack, M. Ornatska, M. Pita and E. Katz. J. Am. Chem. Soc. 130 (2008) 4234.

[28]M. Pita, G. Strack, K. MacVittie, J. Zhou and E. Katz. J. Phys. Chem. B 113 (2009) 16071.

[29]V. Privman, G. Strack, D. Solenov, M. Pita and E. Katz. J. Phys. Chem. B 112 (2009) 11777.

[30] V. Privman, M. A. Arugula, J. Halamek, M. Pita, and E. Katz J. Phys. Chem. B 113 (2009), 5301.

[31]D. Melnikov, G. Strack, M. Pita, V. Privman and E. Katz. J. Phys. Chem. B 113 (2009) 10472.

[32] V. Privman, V. Pedrosa, D. Melnikov, M. Pita, A. Simonian and E. Katz. Biosens. Bioelectron. 25 (2009) 695.

[33]V. Privman, J. Halamek, M. A. Arugula, D. Melnikov, V. Bocharova and E. Katz. J. Phys. Chem. B 114 (2010) 14103.

[34]M. Pita, V. Privman, M. A. Arugula, D. Melnikov, V. Bocharova and E. Katz. Phys. Chem. Chem. Phys. 13 (2011) 4507.

[35]I. Tokarev, V. Gopishetty, J. Zhou, M. Pita, M. Motornov, E. Katz and S. Minko. ACS Appl. Mater. Interf. 1 (2009) 532.

[36]M. Motornov, J. Zhou, M. Pita, V. Gopishetty, I. Tokarev, E. Katz and S. Minko. Nano Lett. 8 (2008) 2993.

[37]M. Motornov, J. Zhou, M. Pita, I. Tokarev, V. Gopishetty, E. Katz and S. Minko. 
Small 5 (2009) 817.

[38]M. Pita, M. Krämer, J. Zhou, A. Poghossian, M.J. Schöning, V.M. Fernández and E. Katz. ACS Nano 2 (2008) 2160.

[39]M. Pita, J. M. Abad, C. Vaz-Dominguez, C. Briones, E. Mateo-Martí, J. A. MartínGago, M. P. Morales and V. M. Fernández. J. Coll. Interf. Sci. 321 (2008) 484.

[40]M. Krämer, M. Pita, J. Zhou, M. Ornatska, A. Poghossian, M. J. Schöning and E. Katz. J. Phys. Chem. C 113 (2009) 2573.

[41]C. Taylor, G. Kenausis, I. Katakis and A. Heller, J. Electroanal. Chem. 396 (1995) 511.

[42]G. Kenausis, C. Taylor, I. Katakis and A. Heller, J. Chem. Soc., Faraday Trans. 92 (1996) 4131.

[43]X. Wang, J. Zhou, T. K. Tam, E. Katz and M. Pita, Bioelectrochemistry 77 (2009) 89.

[44]M. Privman, T. K. Tam, M. Pita and E. Katz, J. Am. Chem. Soc. 131 (2009) 1314.

[45]T. K. Tam, M. Ornatska, M. Pita, S. Minko and E. Katz. J. Phys. Chem. C 112 (2008) 8438.

[46]T. K. Tam, J. Zhou, M. Pita, M. Ornatska, S. Minko and E. Katz. J. Am. Chem. Soc. 130 (2008) 10888.

[47]E. Katz and M. Pita. Chem. Eur. J. 15 (2009) 12554.

[48]L. Amir, T. K. Tam, M. Pita, M. M. Meijler, L. Alfonta and E. Katz, J. Am. Chem. Soc. 131 (2009) 826.

[49]T. K. Tam, M. Pita, M. Ornatska and E. Katz, Bioelectrochemistry 76 (2009) 4.

[50]J. Halamek, T. K. Tam, G. Strack, V. Bocharova, M. Pita and E. Katz, Chem. Comm. 46 (2010) 2405.

[51]T. K. Tam, G. Strack, M. Pita and E. Katz, J. Am. Chem. Soc. 131 (2009) 11670.

[52]M. Pita, T. K. Tam, S. Minko, E. Katz, ACS Appl. Interf Mater. 1 (2009) 1166.

[53]V. Bocharova, T. K. Tam, J. Halamek, M. Pita and E. Katz, Chem. Comm. 46 (2010) 2088.

[54]J. H. Dai, A. W. Jensen, D. K. Mohanty, J. Erndt and M. L. Bruening, Langmuir 17 (2001) 931.

[55]M. Motornov, T. K. Tam, M. Pita, I. Tokarev, E. Katz and S. Minko. Nanotechnology 20 (2009) 434006. 
[56]T. K. Tam, M. Pita, M. Motornov, I. Tokarev, S. Minko and E. Katz. Electroanalysis 22 (2010) 35.

[57]T. K. Tam, M. Pita, M. Motornov, I. Tokarev, S. Minko and E. Katz, Adv. Mater. $22(2010) 12863$.

[58]D. Margulies and A. D. Hamilton, J. Am. Chem. Soc. 131 (2009) 9142.

[59]K. M. Manesh, J. Halamek, M. Pita, J. Zhou, T. K. Tam, P. Santhosh, M.-C. Chuang, J. R. Windmiller, D. Abidin, E. Katz and J. Wang. Biosensors and Bioelectronics 24 (2009) 3569.

[60]J. Halamek, V. Bocharova, S. Chinnapareddy, J. R. Windmiller, G. Strack, M.-C. Chuang, J. Zhou, P. Santhosh, G. V. Ramirez, M. A. Arugula, J. Wang and E. Katz, Mol. BioSyst. 6 (2010) 2554.

[61]J. Halamek, J. R. Windmiller, J. Zhou, M.-C. Chuang, P. Santhosh, G. Strack, M. A. Arugula, S. Chinnapareddy, V. Bocharova, J. Wang and E. Katz, Analyst 135 (2010) 2249.

[62] J. Zhou, J. Halámek, V. Bocharova, J. Wang and E. Katz, Talanta 83 (2011) 955.

[63]M. Privman, T. K. Tam, V. Bocharova, J. Halamek, J. Wang, and E. Katz. ACS Appl. Mat. Interf. 3 (2011) 1620. 


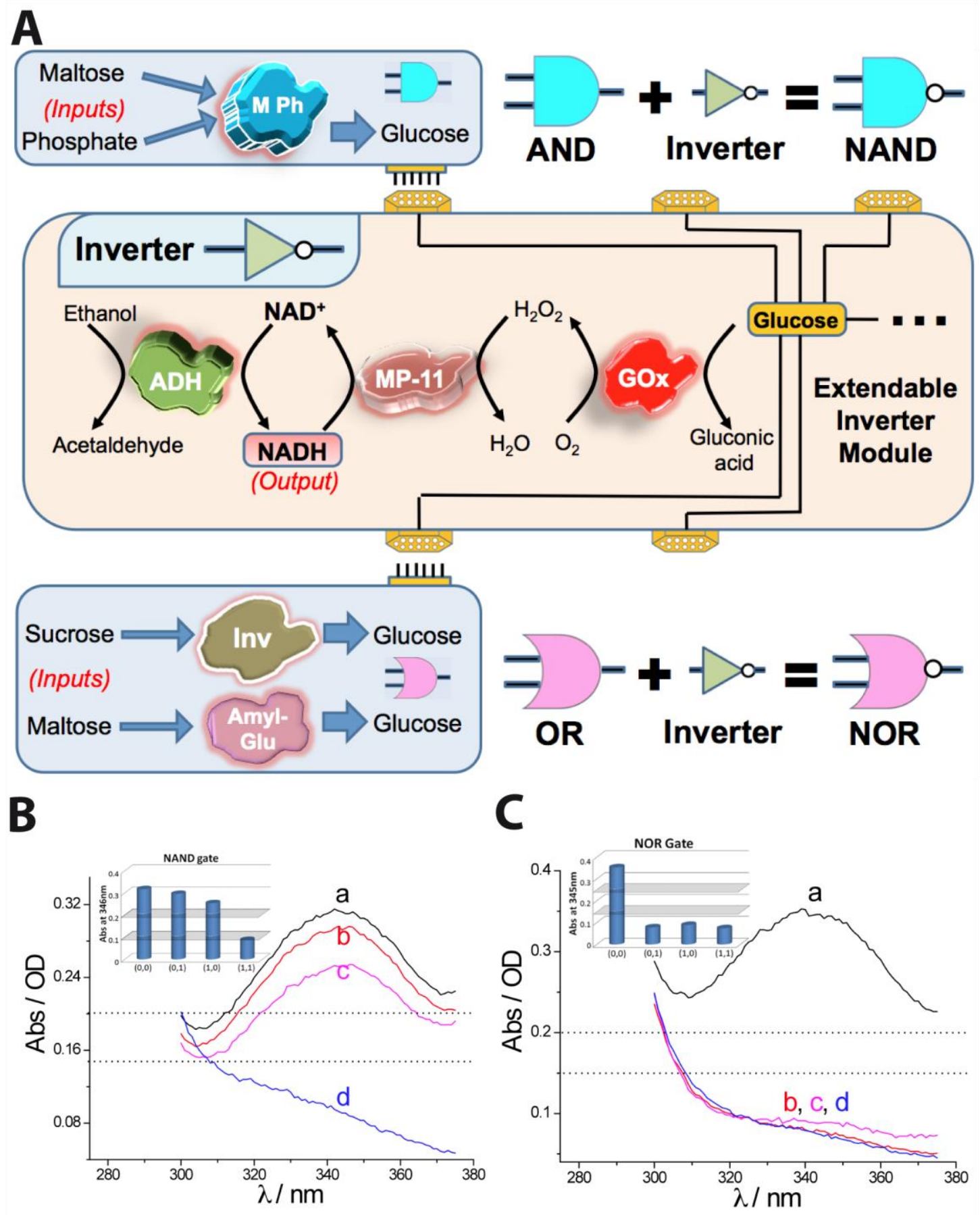

Figure 1. 


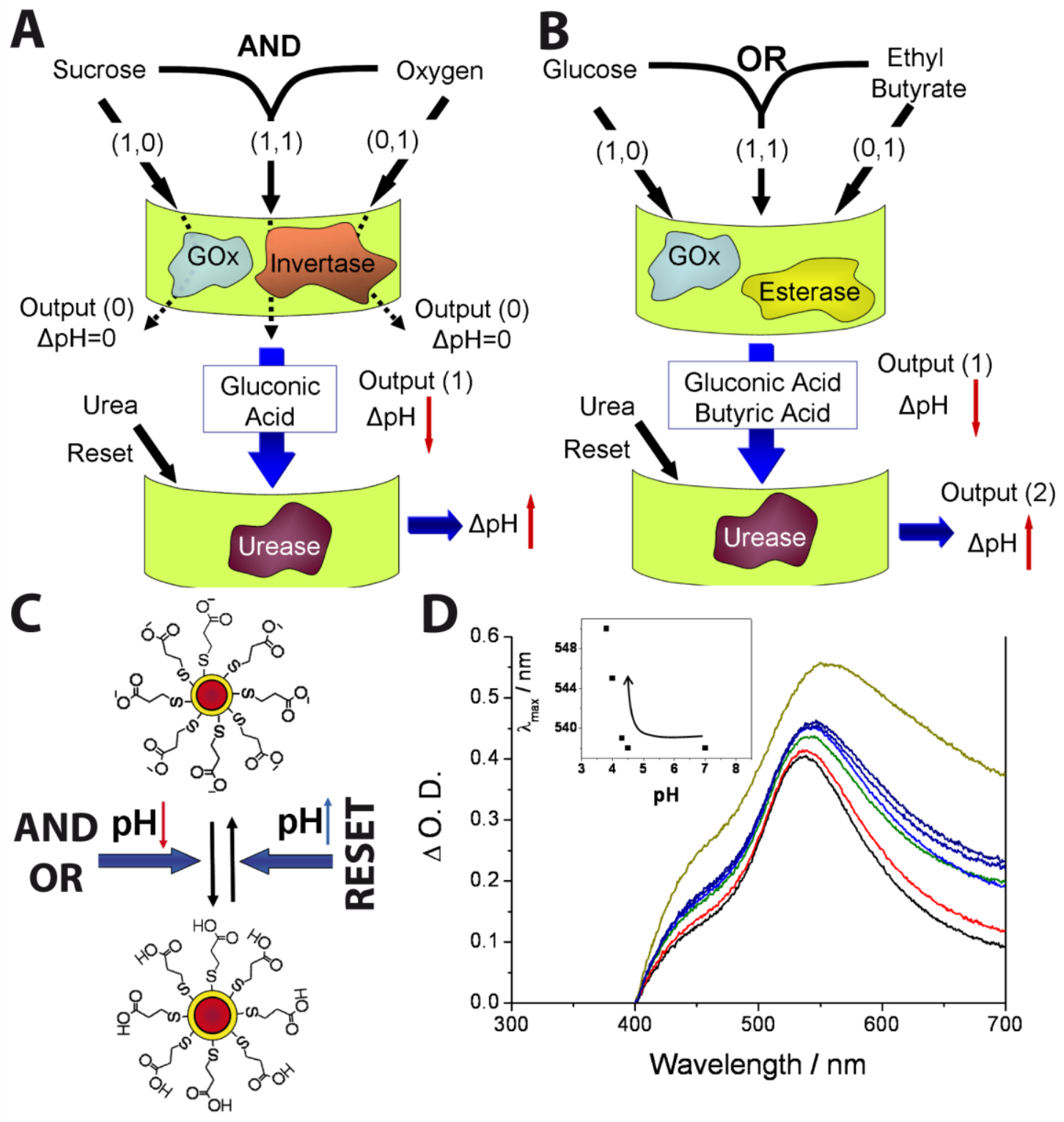

Figure 2. 


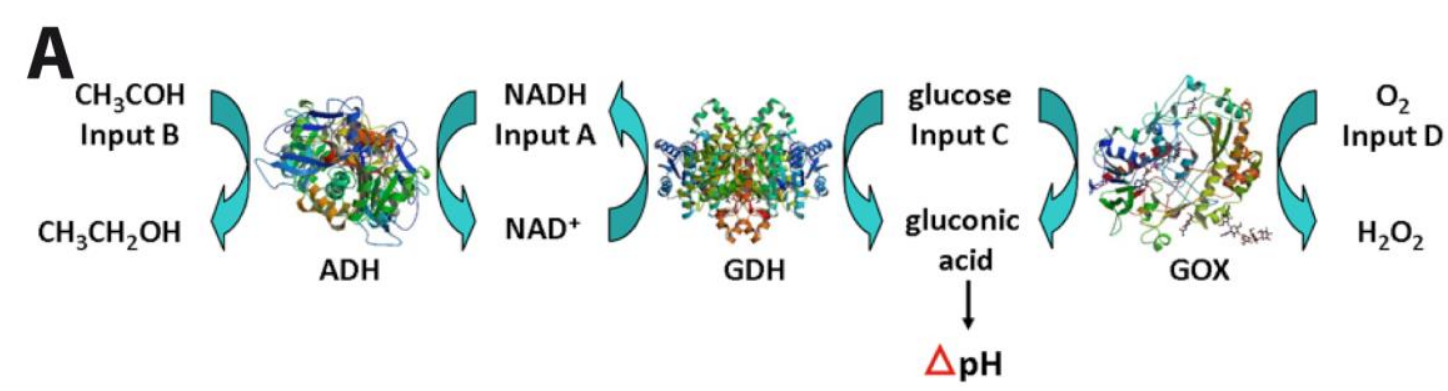

B
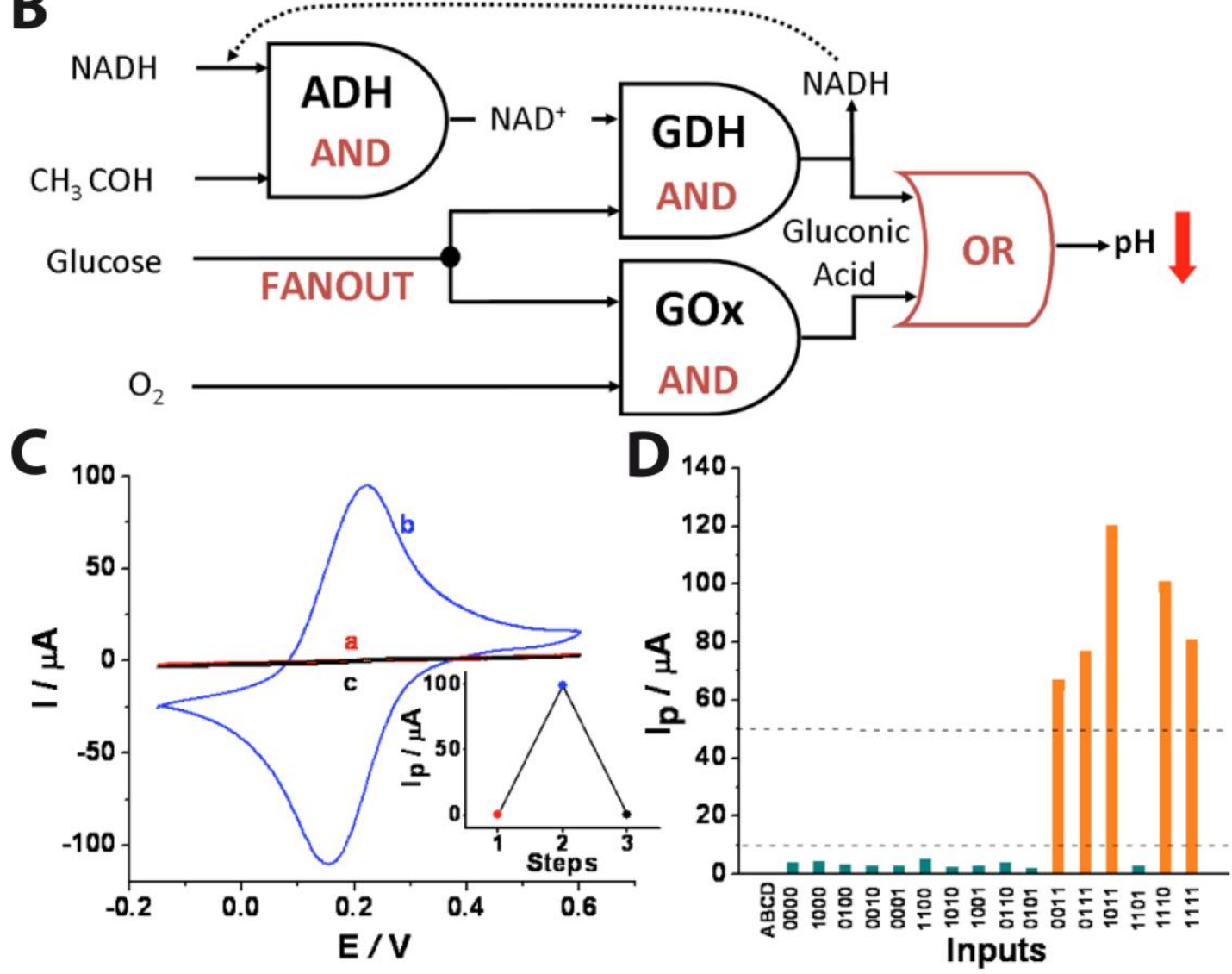

Figure 3. 

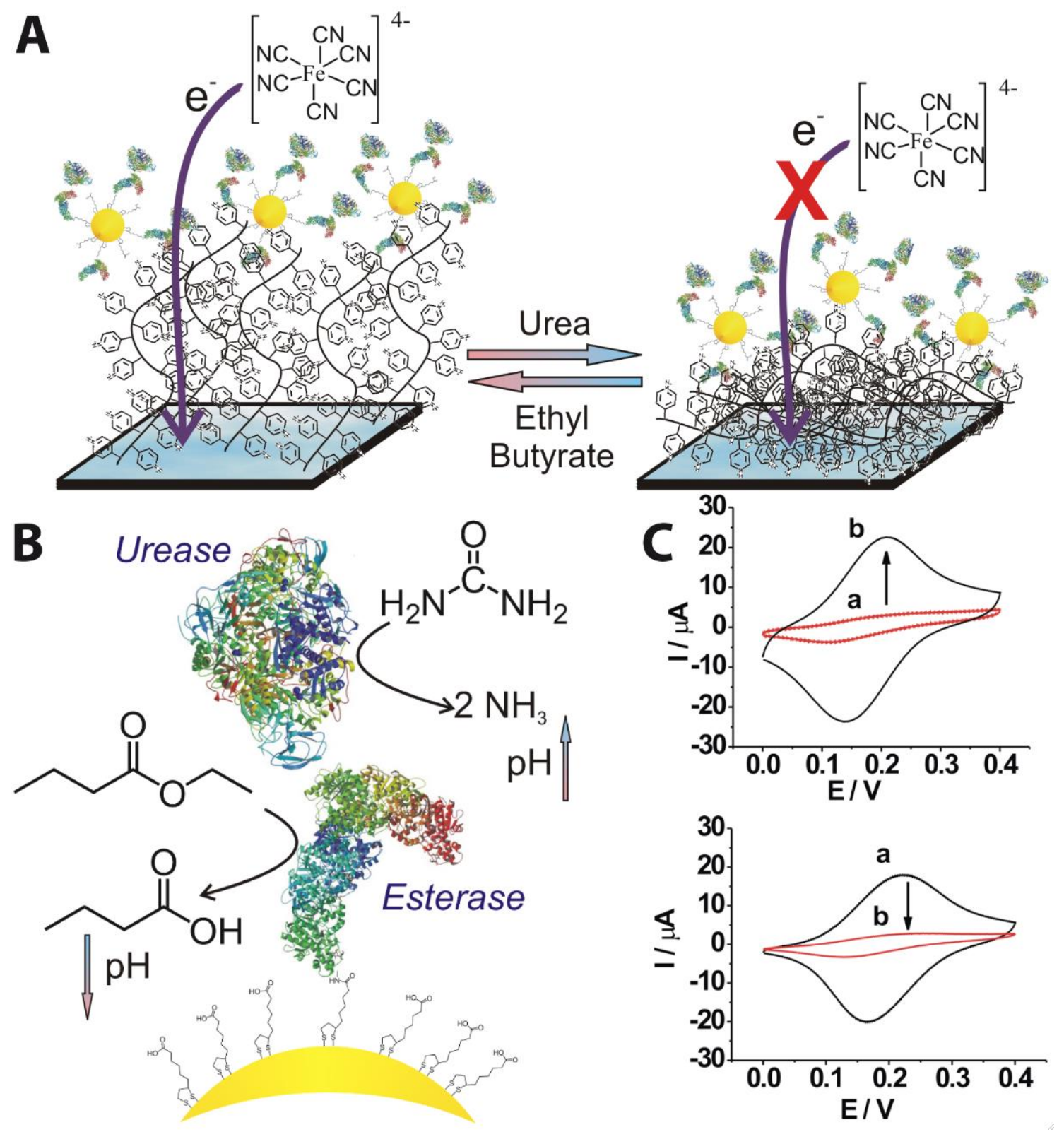

Figure 4. 

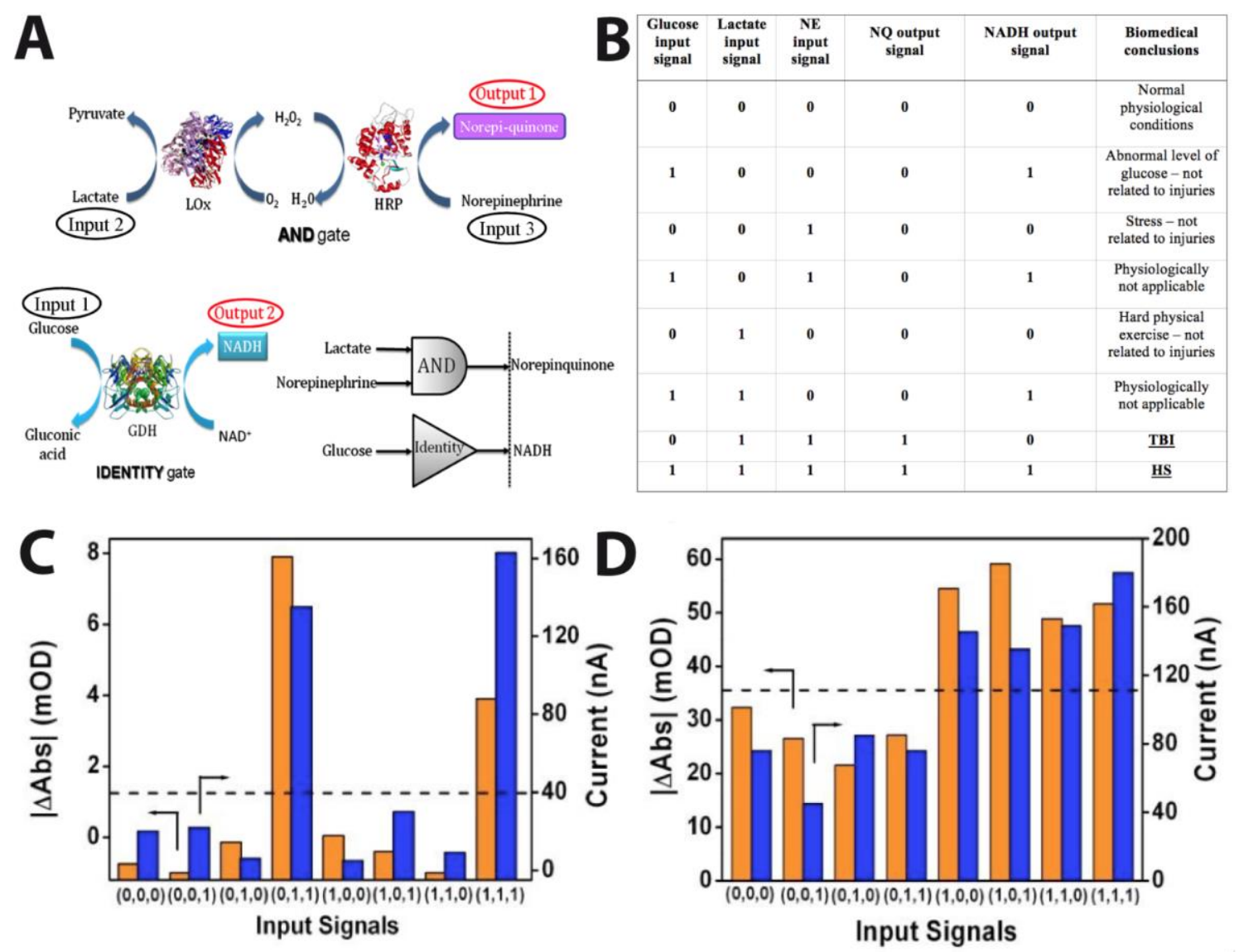

Figure 5. 


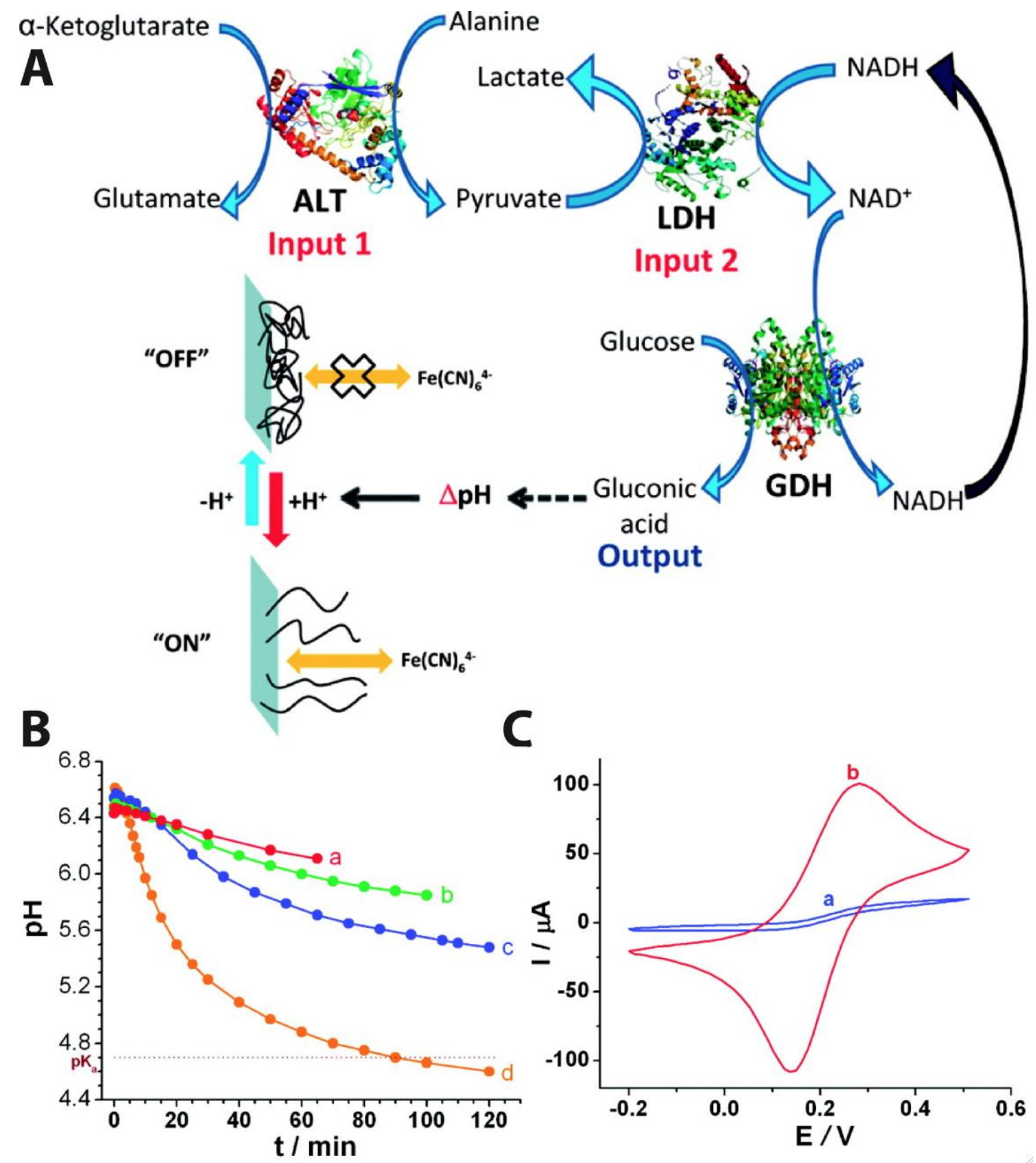

Figure 6. 


\begin{tabular}{|c|c|c|}
\hline \multicolumn{3}{|c|}{ AND } \\
\hline INPUT A & INPUT B & OUTPUT \\
\hline 0 & 0 & 0 \\
\hline 0 & 1 & 0 \\
\hline 1 & 0 & 0 \\
\hline 1 & 1 & 1 \\
\hline
\end{tabular}

\begin{tabular}{|c|c|c|}
\hline \multicolumn{3}{|c|}{ OR } \\
\hline INPUT A & INPUT B & OUTPUT \\
\hline 0 & 0 & 0 \\
\hline 0 & 1 & 1 \\
\hline 1 & 0 & 1 \\
\hline 1 & 1 & 1 \\
\hline
\end{tabular}

\begin{tabular}{|c|c|c|}
\hline \multicolumn{3}{|c|}{ XOR } \\
\hline INPUT A & INPUT B & OUTPUT \\
\hline 0 & 0 & 0 \\
\hline 0 & 1 & 1 \\
\hline 1 & 0 & 1 \\
\hline 1 & 1 & 0 \\
\hline
\end{tabular}

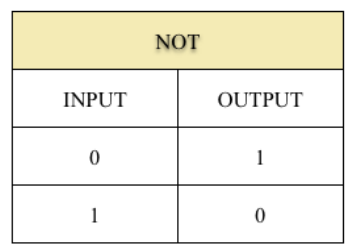

\begin{tabular}{|c|c|c|}
\hline \multicolumn{3}{|c|}{ NAND } \\
\hline INPUT A & INPUT B & OUTPUT \\
\hline 0 & 0 & 1 \\
\hline 0 & 1 & 1 \\
\hline 1 & 0 & 1 \\
\hline 1 & 1 & 0 \\
\hline
\end{tabular}

\begin{tabular}{|c|c|c|}
\hline \multicolumn{3}{|c|}{ NOR } \\
\hline INPUT A & INPUT B & OUTPUT \\
\hline 0 & 0 & 1 \\
\hline 0 & 1 & 0 \\
\hline 1 & 0 & 0 \\
\hline 1 & 1 & 0 \\
\hline
\end{tabular}

\begin{tabular}{|c|c|c|}
\hline \multicolumn{3}{|c|}{ InhA } \\
\hline INPUT A & INPUT B & OUTPUT \\
\hline 0 & 0 & 0 \\
\hline 0 & 1 & 1 \\
\hline 1 & 0 & 0 \\
\hline 1 & 1 & 1 \\
\hline
\end{tabular}

\begin{tabular}{|c|c|c|}
\hline \multicolumn{3}{|c|}{ InhB } \\
\hline INPUT A & INPUT B & OUTPUT \\
\hline 0 & 0 & 0 \\
\hline 0 & 1 & 0 \\
\hline 1 & 0 & 1 \\
\hline 1 & 1 & 1 \\
\hline
\end{tabular}

Table 1. 